\title{
Femoral Pseudoaneurysm as a Complication of Infective Endocarditis
}

\author{
Arjun Gupta, MD, Samar Harris, MD, and Harris Naina, MD
}

University of Texas Southwestern Medical Centre, Dallas, TX, USA.

KEY WORDS: infectious disease; cancer; clinical image.

J Gen Intern Med 31(4):447-8

DOI: $10.1007 / \mathrm{s} 11606-015-3442-\mathrm{z}$

(c) Society of General Internal Medicine 2015

\section{CASE}

A 65-year-old man with chronic lymphocytic leukemia (CLL) presented with 8 months of fatigue and weight loss, and 2 weeks of right groin discomfort. Examination revealed a tender pulsatile right groin mass with a faint bruit. A $\mathrm{CT}$ angiogram revealed a pseudoaneurysm arising from the right proximal deep femoral artery (Figs. 1 and 2). The patient recalled no recent trauma, catheterization, infection, or surgery, but reported a dental extraction 10 months prior. An echocardiogram showed a mitral valve vegetation with severe mitral regurgitation, and blood cultures grew penicillin-sensitive Lactobacillus. A diagnosis of subacute infective endocarditis with mycotic pseudoaneurysm was made. The patient underwent surgical repair of the pseudoaneurysm and mitral valve, and received parenteral antibiotic therapy.

CLL is associated with immunodeficiency, and infection remains the most common cause of death in these patients. ${ }^{1}$ Routine antimicrobial prophylaxis for the prevention of endocarditis is no longer recommended for all patients undergoing dental procedures, although this may be associated with a rise in the rates of endocarditis. $^{2}$ The patient likely developed subacute endocarditis after the dental procedure, with septic emboli leading to vascular wall infection. Extracranial mycotic aneurysms usually present as pseudoaneurysms, and treatment consists of antibiotic and aggressive surgical therapy, including vascular reconstruction as needed. ${ }^{3}$

Received May 5, 2015

Revised May 27, 2015

Accepted June 4, 2015

Published online June 25, 2015
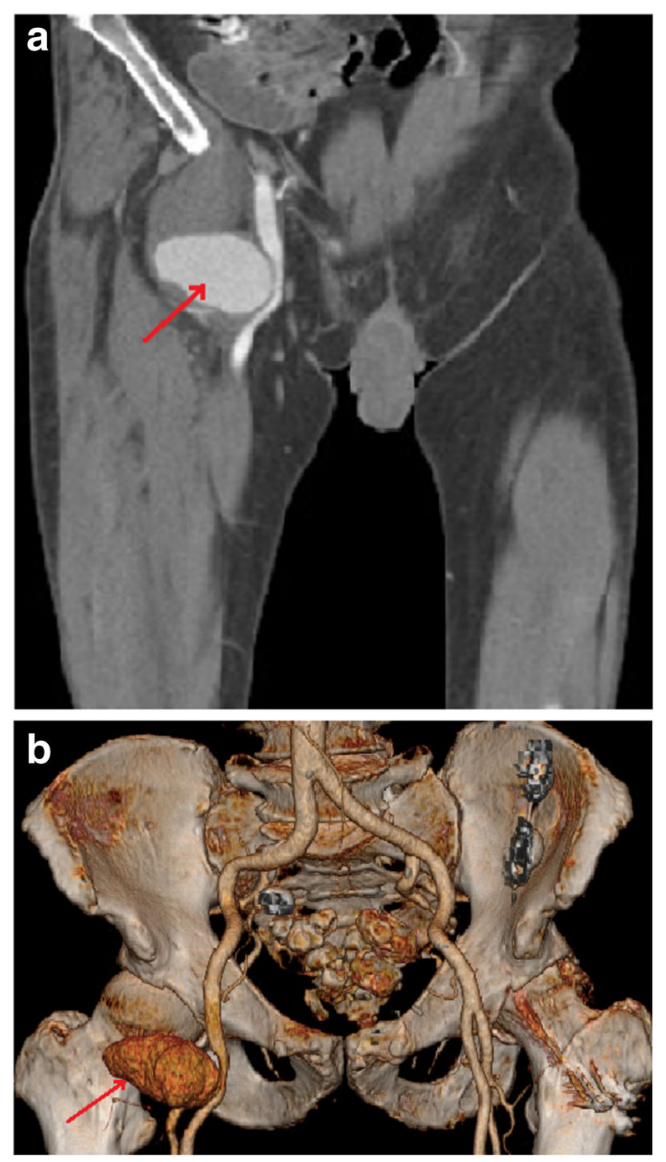

Figs. 1 and 2 CT angiogram (coronal view) demonstrating a mass in the right groin region (a, red arrow), better characterized as a saccular, lobulated pseudoaneurysm measuring $4.1 \mathrm{~cm} \times 5.8 \mathrm{~cm} \times$ $4.1 \mathrm{~cm}$ and arising from the proximal deep femoral artery on threedimensional reconstruction (b, red arrow)

Acknowledgments: All authors have had access to all data in the study, and have read and approved submission of the manuscript. The findings have not been presented previously in any conference or published in print or electronic format.

Funding sources: There are no funding sources, internal or external.

Conflict of Interest: The authors declare that they do not have a conflict of interest.

Corresponding Author: Arjun Gupta, MD; University of Texas Southwestern Medical Centre, 5323 Harry Hines Blvd, Dallas, TX 75390-9047, USA (e-mail: guptaarjun90@gmail.com). 


\section{REFERENCES}

1. Ravandi F, O'Brien S. Immune defects in patients with chronic lymphocytic leukemia. Cancer Immunol Immunother. 2006;55:197-209.

2. Dayer MJ, Jones S, Prendergast B, Baddour LM, Lockhart PB, Thornhill MH. Incidence of infective endocarditis in England, 2000-
13: a secular trend, interrupted time-series analysis. Lancet. 2015;385:1219-28.

3. Reddy DJ, Shepard AD, Evans JR, Wright DJ, Smith RF, Ernst CB. Management of infected aortoiliac aneurysms. Arch Surg. 1991;126:873-8. discussion 8-9. 